Military Technical College Kobry El-Kobbah, Cairo, Egypt

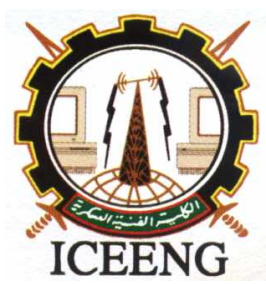

\author{
$6^{\text {th }}$ International Conference \\ on Electrical Engineering \\ ICEENG 2008
}

\title{
Application of the genetic algorithm to design an optimal PID controller for the AVR system
}

By
K. H. Gharib*
O. S. Ebrahim ${ }^{* *}$
H. K. Temraz ${ }^{* *}$
M. A. Awadallah ${ }^{* * *}$

\section{$\underline{\text { Abstract: }}$}

In this paper, a method for optimal proportional-integral-derivative (PID) controller design is proposed to improve the performance of the turbo generator automatic voltage regulator (AVR). The method utilizes the genetic algorithm (GA) to find the controller gains that minimize a synthesized performance index. The performance index considers the system response to both set point and voltage disturbance. Furthermore, the closed loop robust stability is guaranteed by imposing a finite bound on the maximum sensitivity function. Simulation tests are provided to validate the effectiveness of the proposed scheme.

\section{Keywords:}

Proportional-Integral-Derivative (PID) Controller, Genetic Algorithm (GA), Automatic Voltage Regulator (AVR).

\footnotetext{
Ministry of Electricity, Cairo, Egypt

** Faculty of Engineering, Ain Shams University, Cairo, Egypt

*** Faculty of Engineering, Zagazig University, Cairo, Egypt
} 


\section{Introduction:}

The automatic voltage regulator (AVR) of the synchronous generator is responsible for controlling the terminal voltage and reactive power in addition to ensuring proper share of the reactive power amongst parallel connected generators. In power systems, the generators are continuously subjected to load variations and the AVR must cope with normal as well as fault conditions of operation. Such operating condition variations cause considerable changes in the system dynamics. Response variations can in some circumstances cause the AVR to introduce negative damping that can degrade the system stability [1][2].

In order to improve the performance of the AVR system, PID controller is normally used since it has simple structure. Besides, it is robust to variations of the system parameters. The voltage control system of the synchronous generator is of high order and non-linear and therefore; optimal design of the coefficients of this controller is a difficult task. To overcome this difficulty, many methods such as neural network, fuzzy logic, genetic algorithm (GA), and adaptive control techniques have been presented over the past decades [3]-[8]. The advantage of GA technique for tuning the controller parameters is that it is independent of the complexity of the considered performance index. As well as, it suffices to specify an appropriate objective function and to place finite bounds on the optimized parameters [4].

In this paper, we utilize the GA to optimize the performance of the AVR system that it is controlled by PID controller. The performance index developed for tuning the controller considers both the system response to abrupt changes in the set-point and voltage input disturbance. Besides, the robust stability of the closed loop system is guaranteed by specifying finite bound on the maximum sensitivity function. In the following, the proposed method will be introduced.

\section{AVR Model:}

The role of an AVR is to regulate the output voltage of the generator in a specified range. A simple AVR consists of amplifier, exciter, generator and sensor. The block diagram of AVR with PID controller is shown in Figure (1).

The linearized models of the AVR system elements are given in equations (1)-(4) [1].

Amplifier model:

$$
\frac{V_{R}}{V_{E}}=\frac{K_{A}}{1+\tau_{A} s}
$$


Exciter model:

$$
\frac{V_{F}}{V_{R}}=\frac{K_{E}}{1+\tau_{E} s}
$$

Generator model:

$$
\frac{V_{G}}{V_{F}}=\frac{K_{G}}{1+\tau_{G} s}
$$

Sensor model:

$$
\frac{V_{s}}{V_{G}}=\frac{K_{s}}{1+\tau_{s} s}
$$

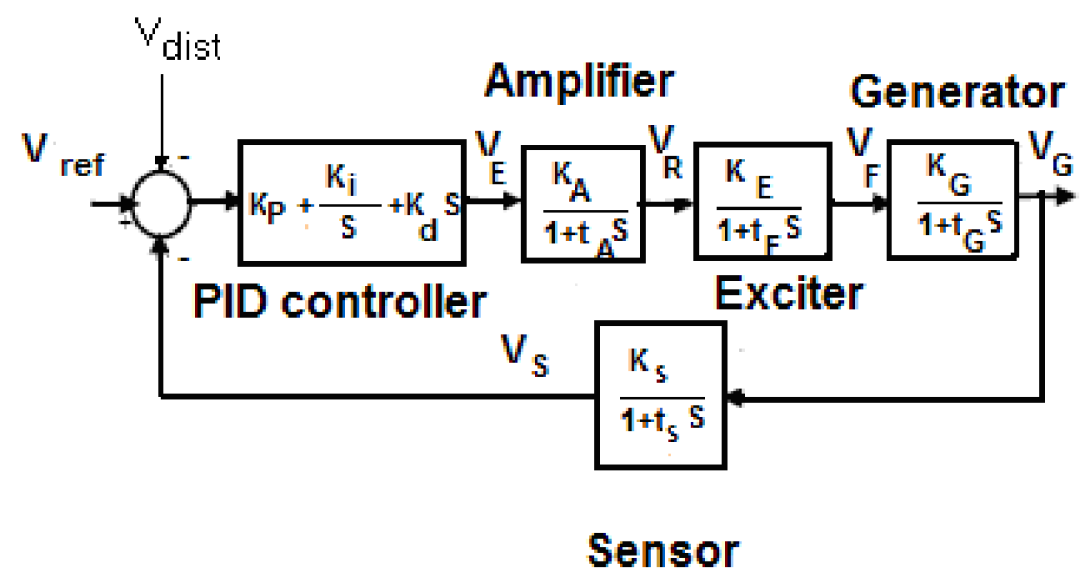

Figure (1): Block diagram of AVR with PID controller.

\section{Problem Formulation:}

Let the PID controller be implemented as

$$
\begin{aligned}
& \frac{V_{E}}{e}=G_{c}(s)=\left(K_{p}+\frac{K_{i}}{s}+K_{d} s\right) \\
& e=V_{r e f}-V_{s}
\end{aligned}
$$

Where $\mathrm{V}_{\mathrm{E}}, \mathrm{V}_{\text {ref }}, \mathrm{V}_{\mathrm{s}}$, and $\mathrm{V}_{\text {dist }}$ are the controller output, set-point, measured output, and input disturbance, respectively. While $\mathrm{K}_{\mathrm{p}}, \mathrm{K}_{\mathrm{i}}$, and $\mathrm{K}_{\mathrm{d}}$ are the proportional, integral, and 
derivative gain of the controller, respectively. Suppose the transfer function of the process is $\mathrm{G}_{\mathrm{p}}(\mathrm{s})$. A measure for the robust stability of the closed loop system can be defined as:

$$
M_{s}=\max _{\omega}\left|\frac{1}{1+G_{p}(j \omega) G_{c}(j \omega)}\right|
$$

Typical value for the maximum sensitivity, $\mathrm{M}_{\mathrm{s}}$, is in the range of 1.4 to 2 [9].

Let $e_{s}$ and $e_{d}$ denote the error caused by unit step set-point change and unit step disturbance at the process input, respectively.

Define the integrated absolute errors as

$$
J_{s}=\int\left|e_{s}\right| d t
$$

and

$$
J_{d}=\int \mid e_{d} d t
$$

In this work, the PID controller is designed such that the performance index,

$$
J=J_{s}+J_{d}
$$

is minimized under the constraint

$$
1.4 \leq M_{s} \leq 2
$$

Clearly, this is a nonlinear optimization problem.

\section{Genetic Algorithm:}

In this paper, the genetic algorithm (GA) is used to solve the PID controller design problem that is formulated in section 3 . The GA is a stochastic optimization algorithm that was originally motivated by the mechanisms of natural selection of evolutionary genetics, and has found many applications in solving global optimization problems. The GA is an alterative procedure that maintains a constant size population of individuals. Each individual represents a potential solution to the problem. Each individual is evaluated to give some measure of its fitness. Some individuals undergo stochastic transformations by means of genetic operation to form new individuals. There are two types of genetic operations: Crossover, which creates new individuals by combining parts from two individuals, and mutation, which creates new individuals by making 
changes in a single individual. The new individuals, called offspring, are then evaluated. New population is formed by selecting fitter individuals from the parent and the offspring population. After several generations, the algorithm converges to the best individuals, which hopefully represents an optimal or suboptimal solution to the problem.

A general procedure of genetic algorithm is given as follows:

1. Generate a population of individual randomly.

2. Evaluate the fitness of each individual in the population.

3. Create offspring by genetic operations.

4. Evaluate the fitness of each offspring.

5. Select new population from parent and offspring population.

6. If the search goal is achieved or maximum allowable generation is attained, stop the search; else go to step (3).

Now, the details of applying GA to the PID controller design will be described.

ENCODING AND INITIALIZATION OF PUPOULATRION: In this work, real number encoding is used to represent the PID controller parameters $K_{p}, K_{i}$, and $K_{d}$ where they are treated as the chromosome of the individual. In most tuning formulas, the parameters of the PID controller stay in the ranges $0<\mathrm{K}_{\mathrm{P}}<\mathrm{K}_{\text {pmax }}, 0<\mathrm{K}_{\mathrm{i}}<\mathrm{K}_{\text {imax }}$, and $0<\mathrm{K}_{\mathrm{d}}<\mathrm{K}_{\mathrm{dmax}}$ [4]. Therefore, the initial population is generated randomly in this range.

FITNESS AND COST FUNCTION: The derived performance index in this study represents a cost function that is function in the PID controller parameters, i.e., $J\left(\mathrm{~K}_{\mathrm{p}}, \mathrm{K}_{\mathrm{i}}\right.$, $\left.\mathrm{K}_{\mathrm{d}}\right)$. Our objective is to search for $\left(\mathrm{K}_{\mathrm{p}}, \mathrm{K}_{\mathrm{i}}, \mathrm{K}_{\mathrm{d}}\right)$ such that $\mathrm{J}$ is minimized under the constraint (10). Thus, an individual that has lower $\mathbf{J}$ should be assigned a larger fitness. Then the GA tries to generate better offspring to improve the fitness. Therefore, optimal PID controller would be corresponding to an individual $\left(\mathrm{K}_{\mathrm{p}}, \mathrm{K}_{\mathrm{i}}, \mathrm{K}_{\mathrm{d}}\right)$ could be defined as

$$
F\left(K_{p}, K_{i}, K_{d}\right)=\frac{1}{J\left(K_{p}, K_{i}, K_{d}\right)}
$$

and $F\left(K_{p}, K_{i}, K_{d}\right)=0$ if the closed loop system is unstable or the maximum sensitivity function $M_{s}$ is outside the range (10).

SELECTION CRITERIA: When doing crossover and mutation operations, the individual that belong to the present population needs to be selected. A commonly used method is the roulette wheel selection. In this method, the jth individual of parent population with fitness value of $F_{j}$ is given a proportional probability $\mathrm{P}_{j}$ of being 
selected according to the distribution [4].

$$
P_{j}=\frac{F_{j}}{\sum F_{j}}
$$

CROSSOVER: The crossover operation comprises three steps. First, two chromosomes in the present population (parents) are chosen by the roulette wheel selection method. Second, randomly take a component from one chromosome to form the corresponding component of the offspring. Third, repeat the second step until the components of the offspring fill up perfectly. The operation of the crossover is shown in Figure (2).

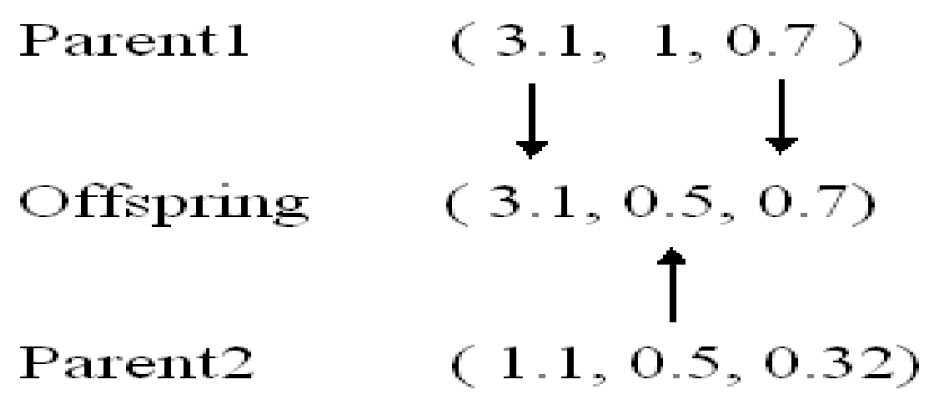

Figure (2): Crossover operation.

MUTATION: Mutation is a way to generate new genetic material to the population. This is done to help the GA avoiding be trapped at a local optimal. For a randomly selected parent $\left(\mathrm{K}_{\mathrm{p}}, \mathrm{K}_{\mathrm{i}}, \mathrm{K}_{\mathrm{d}}\right)$, if $\mathrm{K}_{\mathrm{p}}$ is selected for mutation, the resultant offspring is $\left(\mathrm{K}_{\mathrm{p}}\right.$, $\mathrm{K}_{\mathrm{i}}, \mathrm{K}_{\mathrm{d}}$ ), where $\mathrm{K}_{\mathrm{p}}$ is randomly selected from the following two possibilities [4][8]:

$$
K_{p}^{\prime}=K_{p}+\Delta(i, \lambda), K_{p}^{\prime}=K_{p}-\Delta(i, \lambda) .
$$

The function $\Delta($ i. $\lambda)$ is given as

$$
\Delta(i, \lambda)=r \lambda\left(1-\frac{i}{I}\right)
$$

Where, $\mathrm{r}$ is a random number from o to $1, \mathrm{i}$ is the generation number, $I$ is the maximum number of generation, and $\lambda=\mathrm{K}_{\mathrm{pmax}}$. 


\section{Simulation Results:}

In order to quantify the quality of the proposed GA design algorithm, it is compared with Ziegler-Nichols tuning method [9]. Two AVR systems are simulated having specifications as in Table (1). These parameters are according to the block diagram given in Figure 1.

Table (1): Parameters of the Two Simulated AVR Systems.

\begin{tabular}{|c|c|c|}
\hline Parameter & System-1 & System-2 \\
\hline $\mathrm{K}_{\mathrm{A}}$ & 10 & 40 \\
\hline $\mathrm{T}_{\mathrm{A}}$ & 0.1 & 0.01 \\
\hline $\mathrm{K}_{\mathrm{E}}$ & 1 & 0.2 \\
\hline $\mathrm{T}_{\mathrm{E}}$ & 0.4 & 4 \\
\hline $\mathrm{K}_{\mathrm{G}}$ & 1 & 1 \\
\hline $\mathrm{T}_{\mathrm{G}}$ & 1 & 0.8 \\
\hline $\mathrm{K}_{\mathrm{s}}$ & 1 & 1 \\
\hline $\mathrm{T}_{\mathrm{S}}$ & 0.1 & 0.001 \\
\hline
\end{tabular}

The controller coefficients for system-1 (which is considered here as the nominal system) are found using Ziegler second method of tuning. Figure (3) shows the closed loop system response to unit step change in the reference voltage using Zeigler gains.

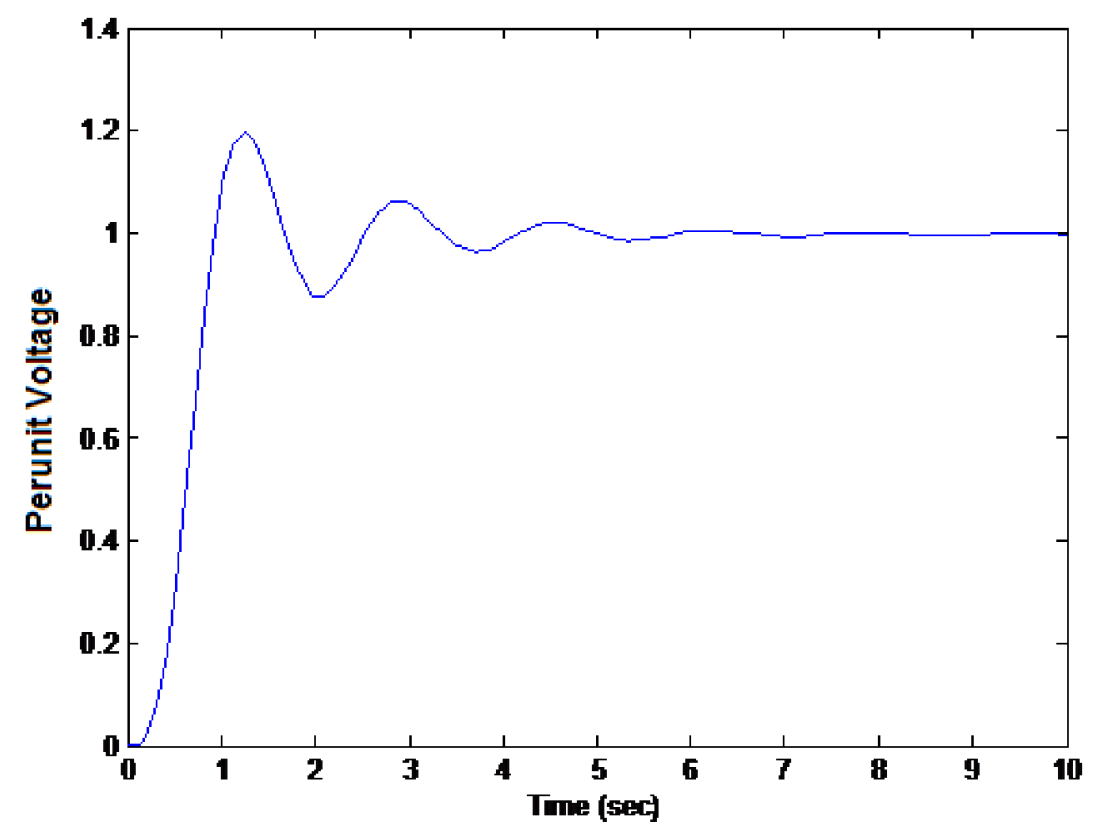

Figure (3): AVR response using Ziegler-tuned PID Controller. 
The PID gains are determined based on the proposed GA using Matlab programming environment. Figure (4) shows the tracking error with generation, where it is clear that it reaches a minimum steady state value after 210 generation. Figure (5) shows the system response to step change in the reference voltage in case of GA-tuned PID controller as well as in case of Ziegler-tuned PID controller. The time domain performance specifications resulting from the two controllers as applied to the AVR system-1 are tabulated in table (2). It is evident that the GA-based tuning method is superior in dynamical performance compared with a PID controller that is tuned using classical method. Figure (6) shows the response to step change in the reference voltage using GA gains when the system parameters equal their nominal value (system-1) and when the parameters vary as in system-2. Where, it is clear that the controller response is nearly insensitive to system parameters variations. Figure (7) shows the response of the AVR system-1 with GA-tuned PID controller (red) and Zeigler-tuned PID controller (blue) under step change of $25 \%$ in the voltage input disturbance $\left(\mathrm{V}_{\text {dist }}\right)$. In that figure, the advantages of having smaller voltage drop and faster attenuation time are clear.

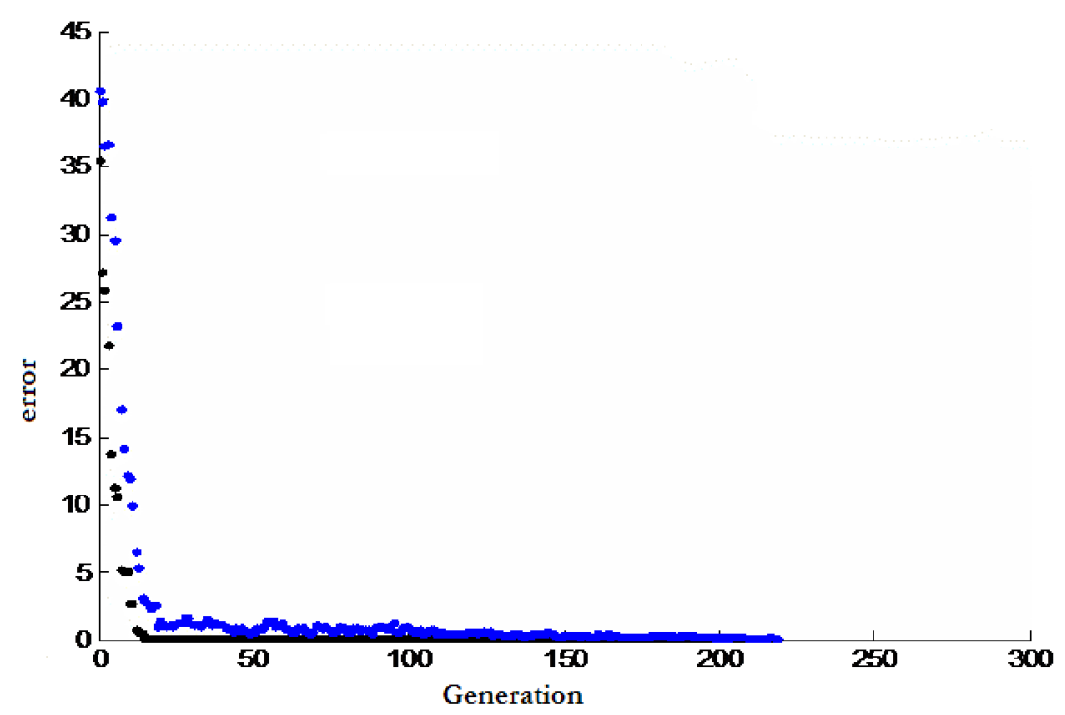

Figure (4): Error verses generation. Average value (blue) and absolute value ( black).

Table (2): Time domain performance specifications.

\begin{tabular}{|c|c|c|}
\hline Parameters & Zeigler-tuned controller & GA-tuned controller \\
\hline Maximum over shoot & $20 \%$ & $2.7 \%$ \\
\hline Settling time & $4.2 \mathrm{~s}$ & $1 \mathrm{~s}$ \\
\hline Rise time & $0.94 \mathrm{~s}$ & $0.46 \mathrm{~s}$ \\
\hline Absolute error & 1.3 & 0.3 \\
\hline
\end{tabular}




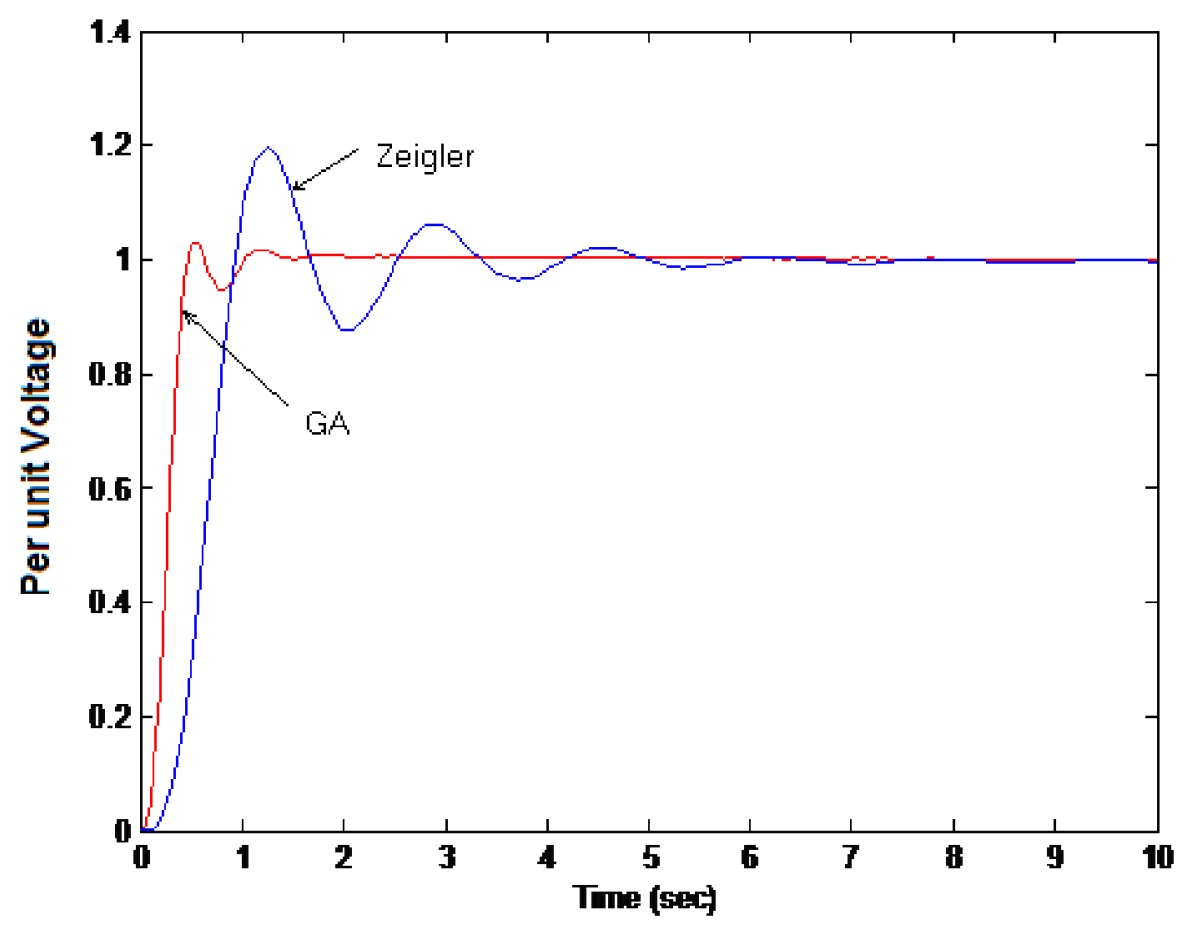

Figure (5): Response of the AVR to step change in the voltage reference.

GA-based PID Controller (red); Zeigler -based PID Controller (blue).

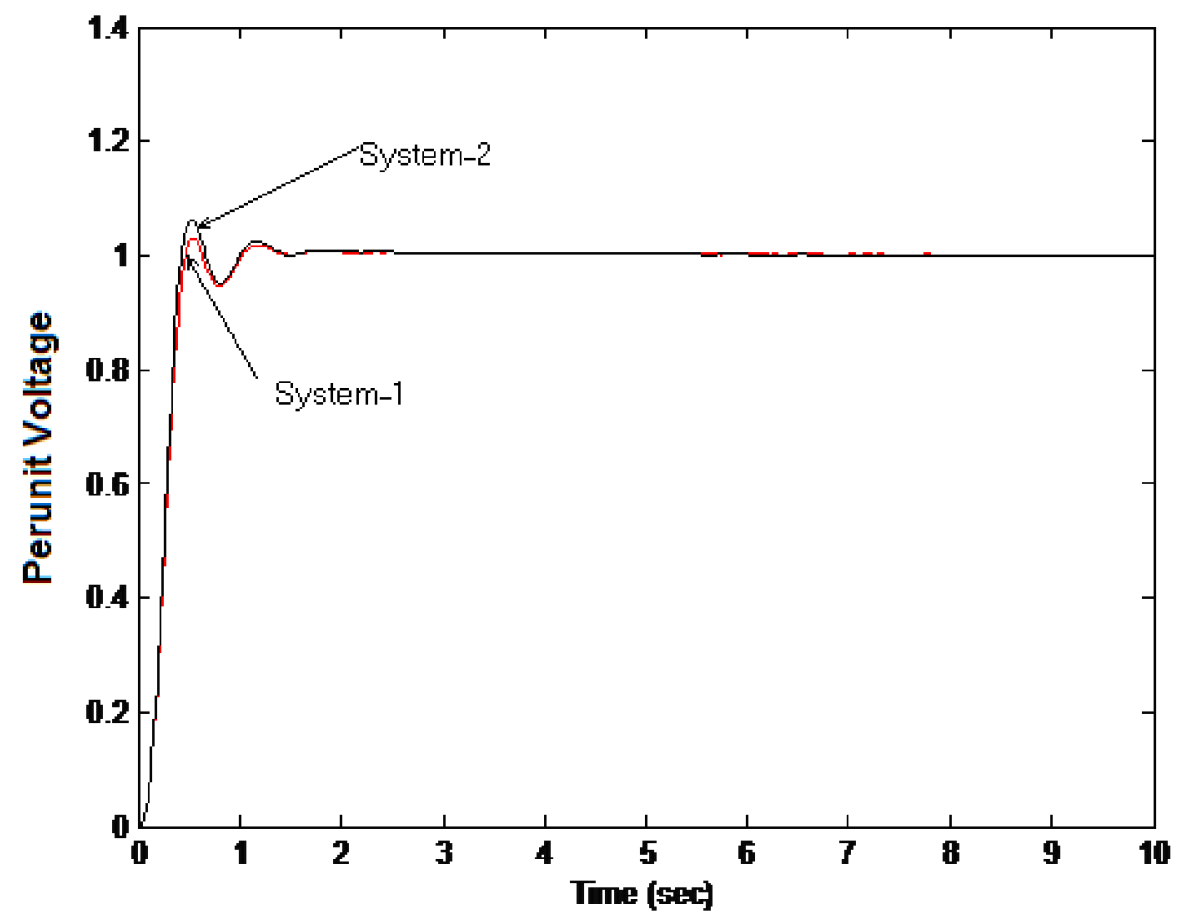

Figure (6): Response to step change in the reference voltage using GA gains for system-1 (red) and system -2 (black). 


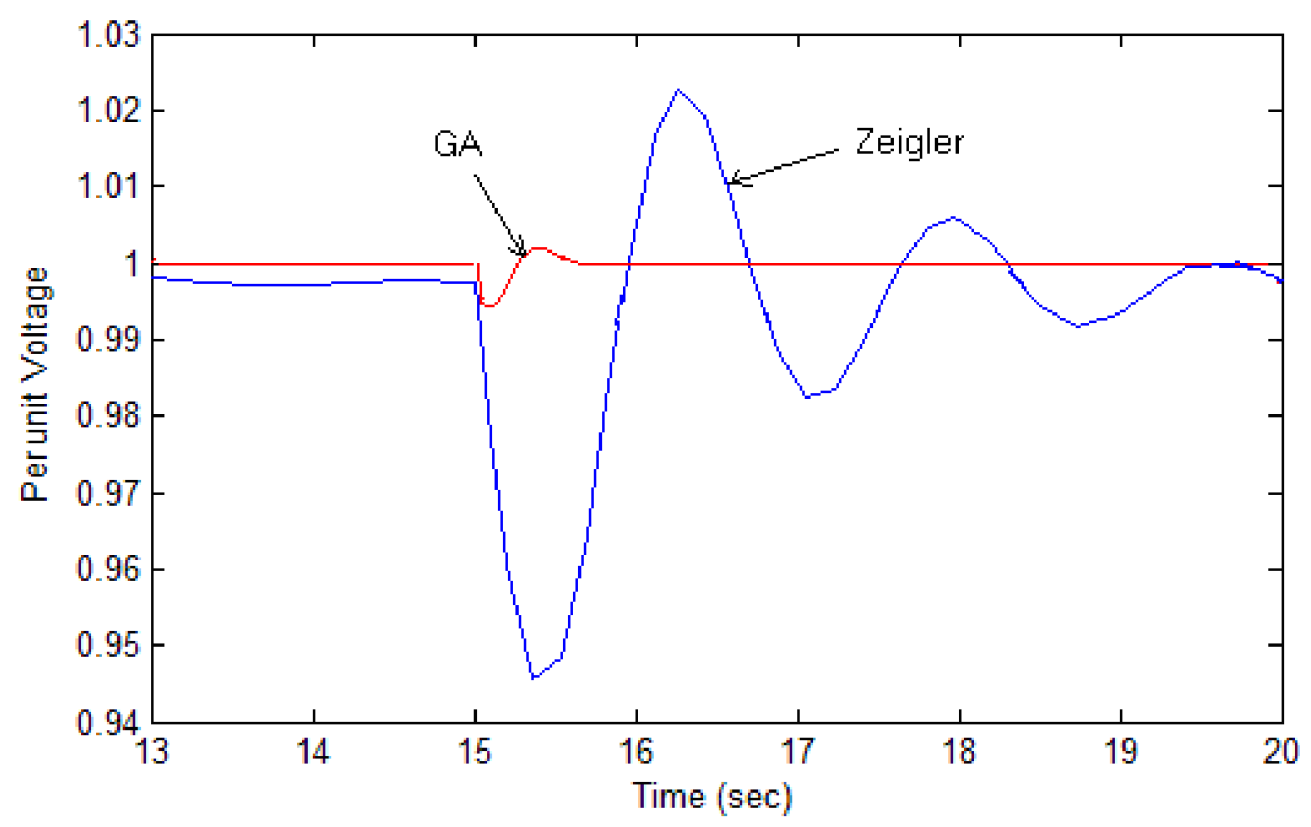

Figure(7): Response of the AVR system-1 under step change of $25 \%$ in the voltage disturbance. GA-tuned PID Controller (red), Zeigler -tuned PID Controller (blue).

\section{Conclusion:}

In this paper, a genetic algorithm for the optimal design of an AVR system with PID controller has been introduced. The cost function of the presented GA considers the setpoint and disturbance responses. Besides, the maximum value of the sensitivity function is defined as a constraint and therefore; the robust stability and performance of the resultant closed loop system are ensured. Simulation studies considering variations in the system parameters and abrupt changes in the set-point and disturbance have been carried out. The obtained results confirmed the effectiveness of the proposed method of tuning.

\section{References:}

[1] Mahdi Banejad and Rahmatlloah Hooshmand Shahrood "Optimal Design of Coefficients of PID Controller in an AVR System using Fuzzy Particle Swarm Optimization Algorithm," AUPEC, 2006. 
[2] EPRI Electric Power Research Institute, "Power Plant Modeling and Parameter Derivation for Power System Studies: Present Practice and Recommended Approach for Future Procedures," Report No. 1015241 USA, June 2007.

[3] R. A. Krohling and J. P. Rey, "Design of Optimal Disturbance Rejection PID Controllers using Genetic Algorithm," IEEE Trans. Evol. Comput., vol. 5, pp. 7882, Feb. 2001.

[4] Y. Mitsukura, T. Yamamoto, and M. Kaneda, "A Design of Self-tuning PID Controllers using a Genetic Algorithm," in Proc. Amer. Contr. Conf., San Diego, CA, pp. 1361-1365, June 1999.

[5] T. Kawabe and T. Tagami, "A Real-Coded Genetic Algorithm for Matrix Inequality Design Approach of Robust PID Controller with Two Degrees of Freedom", in Proc. 12th IEEE Int. Symp. Intell. Contr., Istanbul, Turkey, pp. 119124, July 1997.

[6] A. Visioli, “Tuning of PID Controllers with Fuzzy Logic”, Proc. Inst. Elect. Eng. Contr. Theory Applications, vol. 148, No. 1, pp. 1-8, Jan. 2001

[7] T. L. Seng, M. B. Khalid, and R. Yusof, "Tuning of a Neuro-Fuzzy Controller by Genetic Algorithm”, IEEE Trans. Syst., Man, Cybern., vol. 29, pp. 226-236, Apr. 1999.

[8] R. A. Krohling and J. P. Rey, "Design of Optimal Disturbance Rejection PID Controllers using Genetic Algorithm," IEEE Trans. Evol. Comput., vol. 5, pp. 7882, Feb. 2001.

[9] K. J. Astrom, T. Hagglund, C. C. Hang, and W. H. Ho, " Automatic tuning and adaptation for PID controllers: A survey," IFAC J. Control Engineering Practice, vol. 1, no. 4, pp. 669-714, 1993. 